\title{
Annular Cladding Erbium-Doped Multi-Core Fiber for SDM Amplification
}

\author{
C. Jin ${ }^{1}$, B. Ung ${ }^{2}$, Y. Messaddeq ${ }^{1}$, S. LaRochelle ${ }^{1}$ \\ 1. Centre d'Optique, Photonique et Laser (COPL), Université Laval, Québec, QC, Canada \\ 2. Département de génie électrique, École de Technologie Supérieure, Montréal, QC, Canada \\ sophie.larochelle@gel.ulaval.ca
}

\begin{abstract}
An annular cladding ED-MCF is proposed to enhance pump power efficiency. Simulations suggest amplification with over $29 \mathrm{~dB}$ gain and less than $7.5 \mathrm{~dB}$ noise figure at $1550 \mathrm{~nm}$ using a single Watt-level pump.

OCIS codes: (060.2410) Fibers, erbium; (060.2320) Fiber optics amplifiers and oscillators
\end{abstract}

\section{Introduction}

The phenomenal rise of data traffic, recently driven by the increasing use of video streaming, social media and cloud computing, is expected to saturate the capacity limit of single-mode fibers (SMFs) within a decade or so [1]. Space division multiplexing (SDM) is a promising approach to overcome this anticipated capacity crunch [2] while minimizing the energy per bit [3], [4]. As with current SMF based transmission links, practical SDM links will eventually require relayed amplification between spans. Both in-core [5] and cladding [6], [7] pumped erbium doped multicore fiber amplifiers (ED-MCF) have been reported recently. In-core pumping of an ED-MCF avoids the drawbacks of using multiple EDFAs in parallel, but still requires several single-mode pump lasers as well as spatial channel demultiplexers/multiplexers at the amplifier input. In order to reduce pump complexity and related costs, cladding pumping is actively studied towards efficient multicore amplification. The latter scheme allows the use of a low-cost and high-power multimode laser to pump all cores simultaneously. However, an important limitation to the efficiency of this method is the low pump field overlap with the doped cores.

In this paper, we propose a double-cladding erbium doped 6-core fiber based on an annular-cladding (ACEDMCF) for guiding the pump field. First, we delineate the operating principle of the AC-EDMCF by examining its refractive index profile and outline the design constraints linked to fiber fabrication. Using optimized core parameters, we then investigate the performance of the AC-EDMCF through numerical calculations of the signal gain and noise figure (NF).

\section{Fiber design and parameters}

The fiber cores are placed at the apexes of a hexagon with a pitch $\Lambda=40 \mu \mathrm{m}$. An annular (i.e. "ring") cladding that guides pump light is thus populated by the 6 cores, as shown in Fig. 1(a). The annular cladding (fused silica) is located between a depressed inner cladding (fluorine-doped silica) and the outer cladding (polymer) [Fig. 1(b)].
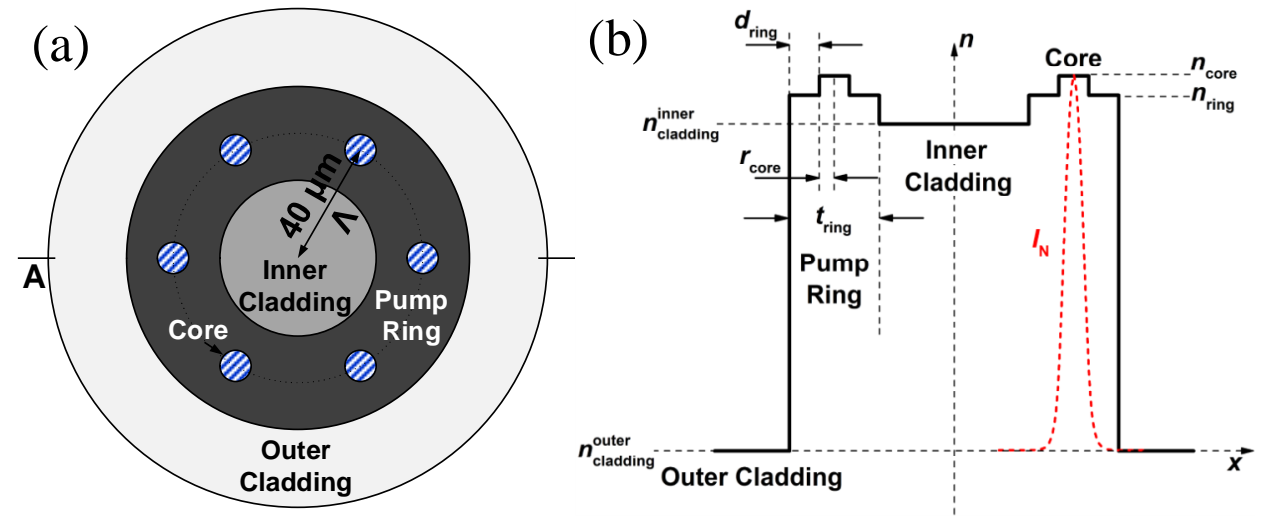

Fig. 1. (a) Transverse cross-section showing the annular pump cladding (dark gray), doped cores (blue), depressed inner cladding (gray), and outer cladding (light gray). (b) Refractive index profile along the $x$ axis (A line in (a)). Red dashed line shows the normalized core signal intensity.

To enhance the pump field overlap with the cores, the annular cladding thickness ( $\left.t_{\text {ring }}\right)$ should be as thin as possible. However, limitations in the fiber fabrication method (preform produced by MCVD and stack-and-draw) require that $t_{\text {ring }} \geq 30 \mu \mathrm{m}$. The refractive index of the depressed inner cladding is also limited by the amount of fluorine 
that can be incorporated in the silica glass giving $n_{\text {inner,clad }}=1.43812$. In our simulations, we considered only one core since all 6 cores are assumed identical. Other assumptions were that background losses are negligible and ionion interactions are ignored $\left(\mathrm{Er}^{3+}\right.$ concentration of $\left.2.62 \times 10^{25} \mathrm{~m}^{-3}\right)$. We also assume that the pump is uniformly distributed in the annular cladding as simulations with beam propagation method showed that this is the case when the pump is injected in the annular cladding using single or multiple spot excitation, as well as via FEM simulations with >200 supported modes. A co-propagating pump source at $980 \mathrm{~nm}$ was utilized and the pump absorption crosssection was taken as $2.18 \times 10^{-25} \mathrm{~m}^{2}$. We performed the simulations with $-30 \mathrm{dBm}$ input signal at $1550 \mathrm{~nm}$ in a $5 \mathrm{~m}$ AC-EDMCF.

We first optimized the core parameters by calculating gain and NF as a function of the signal core radius ( $\left.r_{\text {core }}\right)$ and numerical aperture $(N A)$ using standard coupled rate equations. Figure 2 shows the signal gain over a region limited by the single-mode condition, $V=2.405$ (top right), and low gain condition, $\mathrm{G}<20 \mathrm{~dB}$ (bottom left). As expected, the signal gain increases with core radius since the pump overlap factor can be estimated by $\Gamma_{\mathrm{p}}=A_{\text {core }} / A_{\text {clad. }}$. The NF (not shown), however, slightly increases with the core radius. Keeping within acceptable gain and NF, while enforcing the single mode regime, we choose $r_{\text {core }}=4.5 \mu \mathrm{m}$ and $N A=0.11$ (marked with a solid star in Fig. 2) as the core parameters. An alternate set of parameters $\left(r_{\text {core }}=2.5 \mu \mathrm{m}\right.$ and $\left.N A=0.20\right)$, identified by a solid circle, is used here for comparison purposes. Both sets yield similar overlap factors at $1550 \mathrm{~nm}$, although the former achieves 29.2 $\mathrm{dB}$ small signal gain $(\mathrm{NF}=7.15)$ while the latter gives $25.7 \mathrm{~dB}$ small signal gain $(\mathrm{NF}=7.92)$ using $1 \mathrm{~W}$ pump.

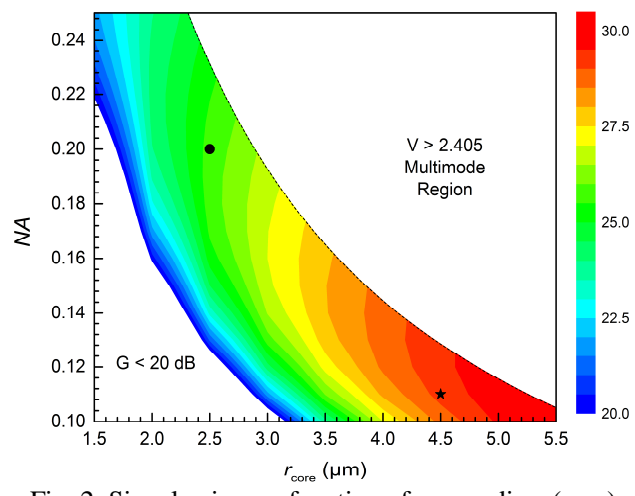

Fig. 2. Signal gain as a function of core radius, $\left(r_{\text {core }}\right)$ and $N A$ for a $1 \mathrm{~W}$ pump.

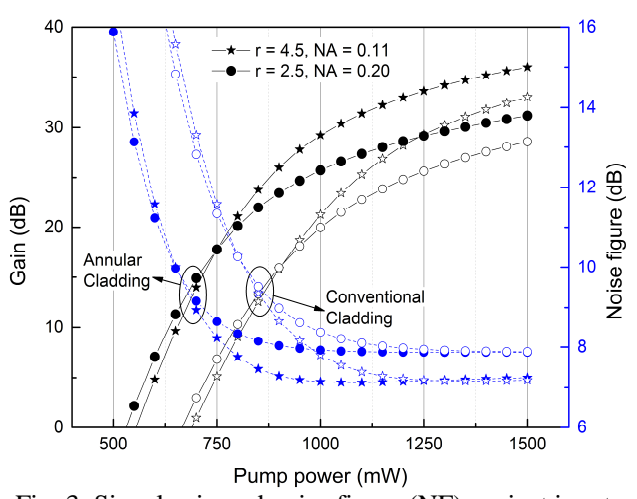

Fig. 3. Signal gain and noise figure (NF) against input pump power from 0.5 to $1.5 \mathrm{~W}$.

\section{Amplification simulation with 6-core AC-EDMCF}

We now inject pump powers from $0.5 \mathrm{~W}$ to $1.5 \mathrm{~W}$ in the annular cladding and calculate the performance for each set of core parameters identified above. Results are shown in Fig. 3 for the proposed AC-EDMCF design (solid markers), while the open markers correspond to a standard double cladding EDMCF (i.e. $n_{\text {inner,clad }}=n_{\text {ring }}$ ). Figure 3 indicates that for a given level of gain, the AC-EDMCF requires significantly less pump power. At all pump power levels, the AC-EDMCF achieves higher gain and lower NF than a similar "ring-less" ED-MCF.

\section{Conclusion}

We propose a 6-core erbium doped fiber with an annular-cladding design for the pump. Comparison with a more conventional "ring-less" multicore amplifier indicates that the proposed design is superior in terms of gain and NF achieved, given similar core parameters. For example, $29 \mathrm{~dB}$ gain is achieved with a $1 \mathrm{~W}$ pump compared to $1.3 \mathrm{~W}$ pump in a standard double-cladding fiber.

\section{References}

[1] R. W. Tkach, "Scaling optical communications for the next decade and beyond, ” Bell Labs Tech. J., vol. 14, no. 4, pp. 3-9, Feb. 2010.

[2] D. J. Richardson, et al., "Space-division multiplexing in optical fibres," Nat. Photonics, vol. 7, no. 5, pp. 354-362, Apr. 2013.

[3] P. J. Winzer, "Energy-Efficient Optical Transport Capacity Scaling Through Spatial Multiplexing, ” IEEE Photonics Technol. Lett., vol. 23, no. 13, pp. 851-853, Jul. 2011.

[4] P. Krummrich et al., "Evaluation of Potential Optical Amplifier Concepts for Coherent Mode Multiplexing, " in Optical Fiber Communication Conference/National Fiber Optic Engineers Conference 2011, 2011, p. OMH5.

[5] K. S. Abedin, et al., "Amplification and noise properties of an erbium-doped multicore fiber amplifier, " Opt. Express, vol. 19, no. 17, pp. 16715-21, Aug. 2011.

[6] K. S. Abedin, et al., "Cladding-pumped erbium-doped multicore fiber amplifier.," Opt. Express, vol. 20, no. 18, pp. 20191 -200, Aug. 2012.

[7] Y. Tsuchida, et al., "Multicore EDFA for Space Division Multiplexing by Utilizing Cladding-pumped Technology, " in Optical Fiber Communication Conference, 2014, p. Tu2D.1. 